\title{
A new Lorentz chaotic system and its circuit implementation
}

\author{
Xingce Liu ${ }^{1}$, Xiaoyu Zhang ${ }^{2}$, Peng Li ${ }^{1}$, , Huizhen Yan $^{1}$ \\ \{lipeng@dlpu.edu.cn\} \\ ${ }^{1}$ School of Information Science and Engineering, Dalian polytechnic University, Dalian, 116034, China \\ ${ }^{2}$ Agronomy College, ShenYang Agricultural University, Shenyang, 110161, China

\begin{abstract}
In this paper, a newfashioned Lorentz chaotic system are found. According to the circuit structure, we set up the dimensionless model of the circuit. In the simulation, three different shapes of chaotic attractors are found. The equilibrium point and stability of chaotic system are analyzed by using the traditional dynamical analysis methods, and the dynamical behaviors with two varying parameters of this circuit are analyzed in detail. In addition, The specific phenomenon of chaotic attractor coexistence is discovered. Finally, the circuit simulation is carried out using Multisim, and the results are compared with the numerical simulation, which proves the validity of the theoretical analysis. Combined with Theoretical analysis and simulation consequence, it is shown that the new chaotic system has abundant dynamic characteristics.
\end{abstract}

Keywords: Memristor, Lorentzian chaotic system, Attractor coexistence, Multisim simulation

\section{Introduction}

Chaos is a kind of motion state that exists universally in nature. It has many characteristics worth studying, such as sensitivity to initial conditions, randomness of change and unpredictability of long term behavior. Macroscopically, although it presents an irregular state, its essence is an orderly movement[1,2].If there is to be chaos in a circuit, there must be nonlinear elements. Memristor can be said to be the most representative element of nonlinear elements. In 1971, Professor Chua projected the existence of memristors are based on the completeness principle of variable combinations[3].In 1976, Professor Chua further elaborated the composition principle and application characteristics of memristors, and explained that memristors can be divided into magneto controlled memristors and charger-controlled memristors[4]. Due to no memory element has been found, memristor research has not received much attention from the scientific and engineering circles. In 2008, HP Labs completed the implementation of memristor, and great progress has been made in the research 
of memristor[5-8].

Lorentz system is familiar to most chaos scholars[9-11]..Lorentzian equation was determined by American meteorologist Lorentz when he simulated the weather, which is an aperiodic phenomenon[12]. The three variables of this equation simulate temperature, humidity and pressure respectively[13]. Based on his results, Lorenz concluded that small initial differences would become larger over time, and based on this, he found that long-term weather forecasting was impossible[14]. This is also regarded as the beginning of the study of nonlinear chaotic theory, so Lorentz system performs an increasingly important role in the research of nonlinear science [15-19]. A newfashioned fifth-order chaotic system is established by combining Lorentz system with a new memristor. The dynamic behavior of Lorentz system combined with the new memristor is studied emphatically.

In this system, a new memristor is introduced on the basis of Lorentz system, and several chaotic attractors are found[20].As is known to all, the dynamical behavior of chaotic circuits containing memristors is closely relevant to the initial state of memristors, which may lead to multi-stability or extremely multi-stability of the system[21-23]. Multistable or extremely multi-stable phenomenon refers to the coexistence of multiple attractors or infinitely many attractors, which is a nonlinear dynamics phenomenon with great research significance [24]. It is a kind of dynamic behavior related to initial conditions, which is often encountered in some nonlinear dynamic systems[25]. Since the system parameters aren't changed and only the initial values are changed, the trajectory of the system can asymptotically approach to a variety of stable states, such as periodic, quasi-periodic, chaotic or hyperchaotic[26]. Multistable or extremely multi-stable nonlinear dynamic systems not only provide great selectivity for their potential engineering applications, but also pose new challenges to control multi-stable states[27].The coexistence behavior of chaotic attractor is found in this system, and the system is discussed deeply[28].

The rest of this article is outlined as follows. In the second section, the model of memristor is introduced. In the third section, the simple chaotic circuit is introduced, the equivalent differential equation of the circuit is introduced, and the dynamic characteristics of the system is analyzed. In the fourth section, the consequence of theoretical analysis are verified on Multisim platform. The fifth section is a summary of the conclusions of this research.

\section{The model of memristor}

Professor Bao has proposed a new type of magneto controlled memristor. This memristor can describe a smooth quadratic nonlinear characteristic curve, and its memristor's model is 
shown as:

$$
\left\{\begin{array}{l}
i=W(\varphi) v \\
\frac{d \varphi}{d t}=v \\
W(\varphi)=-a+b|\varphi|
\end{array},\right.
$$

Thus, the $v-i$ curve can be expressed as:

$$
\left\{\begin{array}{l}
i_{m}=W(\varphi) v_{s} \\
\dot{\varphi}=v_{s}
\end{array},\right.
$$

$W(\varphi)$ in Equation (1) is the relation of magnetic flux $\varphi$. The graph of the function is the characteristic curve that goes through the origin. The slope of the curve is called memorialization, which represents the ratio between charge and magnetic flux. The units of memorialization and conductance are Siemens.Memorific can be expressed as:

$$
\mathrm{W}(\varphi)=\alpha+\beta|\varphi|,
$$

The $\alpha$ and $\beta$ in the equation are the coefficients of the memristor. The magnetic flux of $\Phi$ represents today.

$V_{S}$ is AC power supply in Equation (2), It can be expressed as: $V_{S}=A \sin (2 \pi f t)$, Where $\mathrm{A}$ is the swing of the AC power supply, and $f$ is the frequency of the AC power supply. The sinusoidal AC supply $V_{S}$ is the input of the memristor, $\alpha=0, \beta=1$ in Equation (3). When $A=19$ and the frequency $f$ is $5 \mathrm{~Hz}$ and $50 \mathrm{~Hz}$ respectively, the volt-ampere characteristics of the memristor are shown in Figure 1. When sinusoidal AC excitation is used, the volt-ampere diagram of the memristor is a closed "8" curve through the origin.With the increase of frequency $f$, the area of the side lobe of the curve decreases gradually. This is consistent with the basic properties of memristors. 

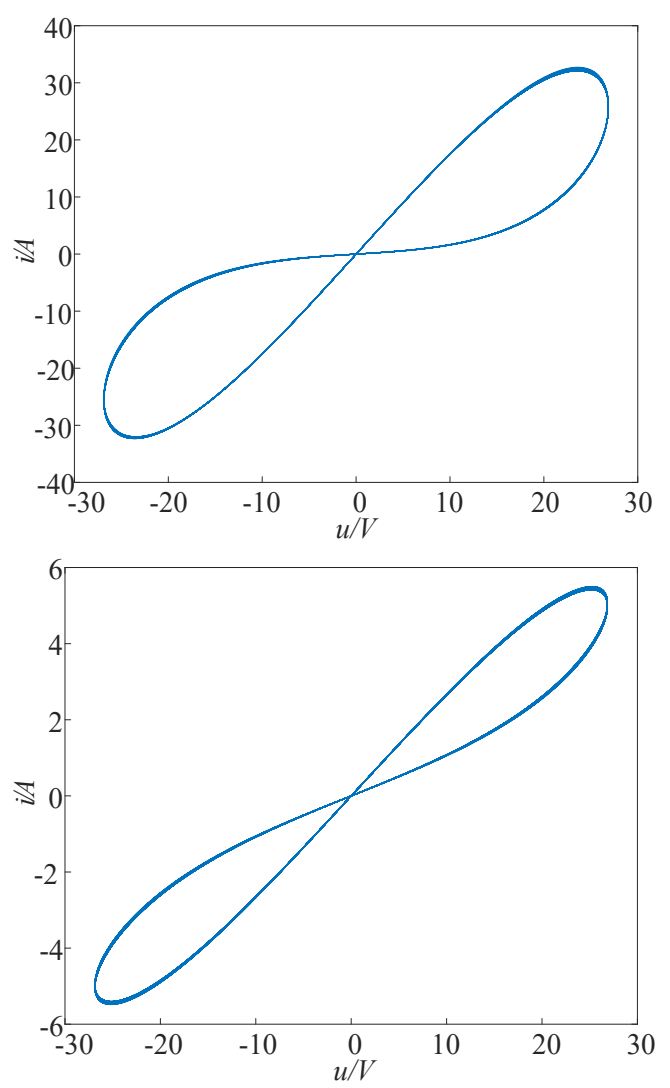

(a) $f=5 \mathrm{~Hz}$

(b) $f=50 \mathrm{~Hz}$

Fig. 1 Hysteresis curve

\section{A memristor-based novel chaotic circuit}

\subsection{A novel chaotic circuit}

The schematic diagram of the novel Lorentz type chaotic circuit is shown in Figure 2 (a), the $\mathrm{G}_{\mathrm{M}}$ in the figure is a new memristor, and its circuit schematic diagram is shown in Figure 2 (b): 


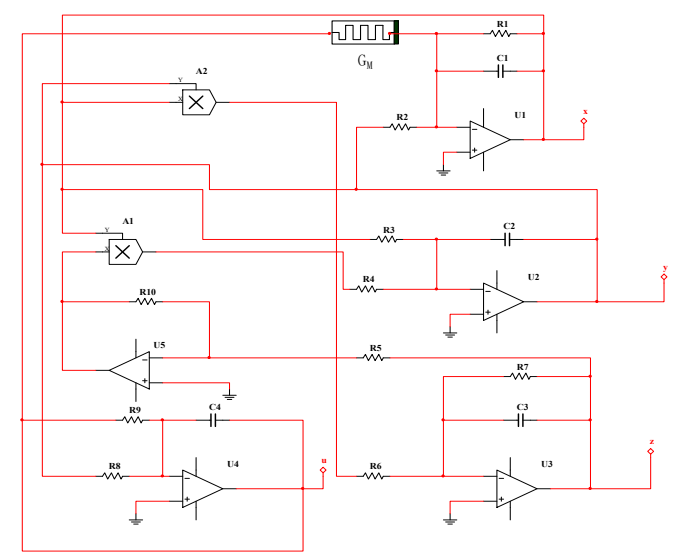

(a)

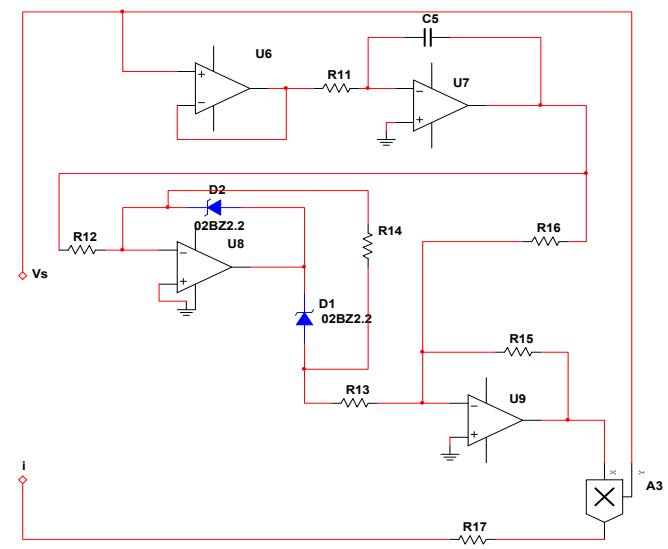

(b)

Fig. 2 (a) the main circuit diagram (b) the memristor circuit

The values of components shown in Fig. 2 circuit are shown in Table 1:

Tab 1. Circuit element value list

\begin{tabular}{cccc}
\hline name & category & numerical & unit \\
\hline$R 1, R 2$, & resistance & 5 & $\mathrm{~K} \Omega$ \\
$R 3$ & resistance & 1.2 & $\mathrm{~K} \Omega$ \\
$R 4$ & resistance & 0.51 & $\mathrm{~K} \Omega$ \\
$R 5, R 7$, & resistance & 10 & $\mathrm{~K} \Omega$ \\
$R 8, R 10$, & resistance & 5 & $\mathrm{~K} \Omega$ \\
$R 11$ & resistance & 50 & $\mathrm{~K} \Omega$ \\
$R 12, R 14$, & resistance & 10 & $\mathrm{~K} \Omega$ \\
$R 16$ & resistance & 10 & $\mathrm{~K} \Omega$ \\
$R 17$ & resistance & 69 & $\mathrm{~K} \Omega$ \\
$C 1, C 2$, & capacitanc & 100 & $\mathrm{nF}$ \\
$C 4, C 5$ & capacitanc & 100 & $\mathrm{nF}$ \\
\hline
\end{tabular}




\begin{tabular}{cccc}
\hline$A 1$ & multiplier & 0.1 & $\backslash$ \\
$A 2, A 3$ & multiplier & 1 & $\backslash$ \\
\hline
\end{tabular}

The ideal magnetron memristor in the figure is composed of voltage follower, reverse integral circuit, absolute value circuit, multiplier and resistance R. its mathematical model can be easily obtained by analyzing the relationship between input voltage and output current. The relationship between output $i$ and input $v_{s}$ can be expressed as follows:

$$
\left\{\begin{array}{l}
i=W\left(v_{w}\right) v_{s}=\frac{g_{1}}{R}\left|v_{w}\right| v_{s}=\frac{k}{R}\left|v_{w}\right| v_{s} \\
\dot{v}_{w}=\frac{v_{s}}{R C_{0}}
\end{array},\right.
$$

Where $V_{w}$ is the internal variable of the ideal magnetron memristor, and $g l$ is the gain of the multiplier $A_{0}$, where $\mathrm{k}=\mathrm{g}_{1} \mathrm{R}_{1} / \mathrm{R}_{2}$, and the memristor $\mathrm{W}\left(v_{w}\right)=\frac{k v_{w}}{R_{1}}$.

According to Kirchhoff's law of voltage and current and the basic characteristics of each element, the system equation can be obtained for the chaotic circuit shown in Figure 2 (a), which is expressed as:

$$
\left\{\begin{array}{l}
R C \frac{d v_{x}}{d t}=-\frac{R v_{x}}{R_{1}}-\frac{R v_{y}}{R_{2}}-\frac{g_{1}}{R_{11}} R\left|v_{w}\right| v_{u} \\
R C \frac{d v_{y}}{d t}=\frac{g_{2} R_{f} R v_{z} v_{x}}{R_{3} R_{4}}-\frac{R v_{x}}{R_{5}} \\
R C \frac{d v_{z}}{d t}=-\frac{g_{3} R v_{x} v_{y}}{R_{6}}-\frac{R}{R_{7}} v_{z} \\
R C \frac{d v_{u}}{d t}=-\frac{R}{R_{8}} v_{y}-\frac{R}{R_{9}} v_{u} \\
R C \frac{d v_{w}}{d t}=\frac{R}{R_{11}} v_{u}
\end{array},\right.
$$

Where $V_{x}, V_{y}, V_{z}, V_{u}$ are four circuit variables, $V_{w}$ is the internal variable of ideal magnetron memristor, $g 1, g 2, g 3$ are the gains of multipliers A1,A2,A3, by dimensionless treatment of the equation, the equation can be expressed as: 


$$
\left\{\begin{array}{l}
\dot{x}=a(x+y)-|w| u \\
\dot{y}=9.8 x z-45 x \\
\dot{z}=-10 x y-c z \\
\dot{u}=b y-5 u \\
\dot{w}=u
\end{array},\right.
$$

When the parameters of system are chosen to be $a=-10, b=-10, c=8$, the initial value are chosen to be $(1,1,0,0,0)$ and the time step is selected 0.01 seconds, the five Lyapunov exponents are expressed as $\mathrm{L} 1=0.6966, \mathrm{~L} 2=0, \mathrm{~L} 3=-0.1671, \mathrm{~L} 4=-2.6321, \mathrm{~L} 5=-20.8969$. The three kinds of different chaotic attractors obtained by simulation can be shown in Figure 3.

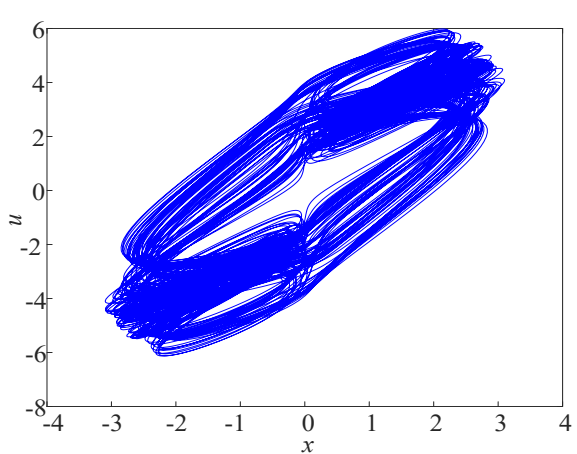

(a) $x-u$

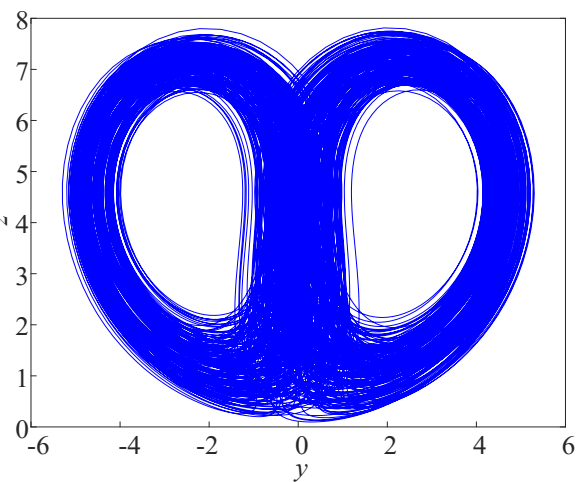

(b) $y-z$

Fig. 3 When $a=-10, b=-10, c=8$, chaotic attractor phase diagram

Since the parameters $a, b, c$ are changed and the initial condition and step size are kept unchanged, the following two kinds of chaotic attractor phase diagrams are obtained:
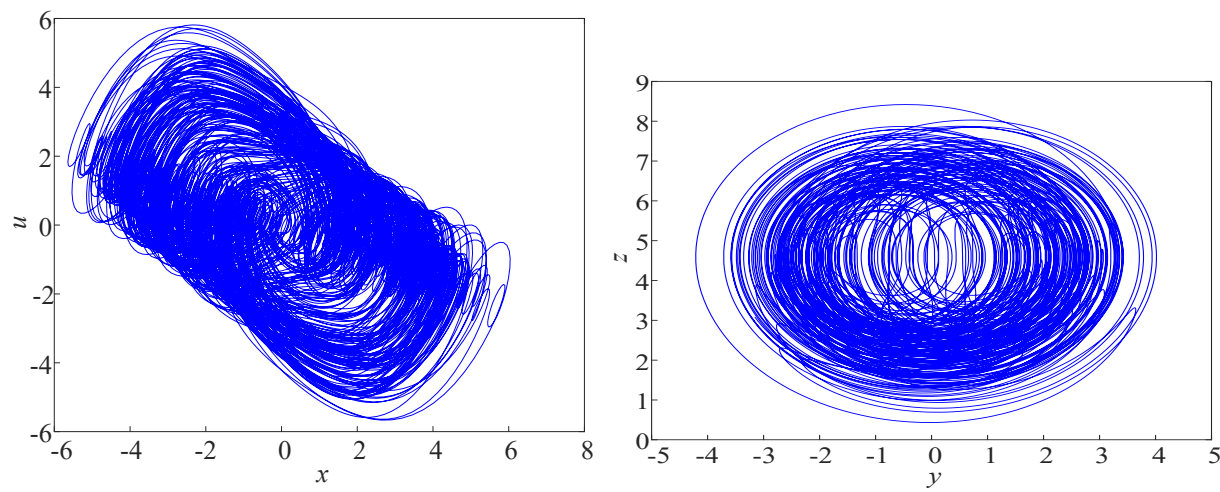
(a) $x-u$

(b) $y-z$

Fig. 4 When $a=-5, b=-15, c=3$, chaotic attractor phase diagram

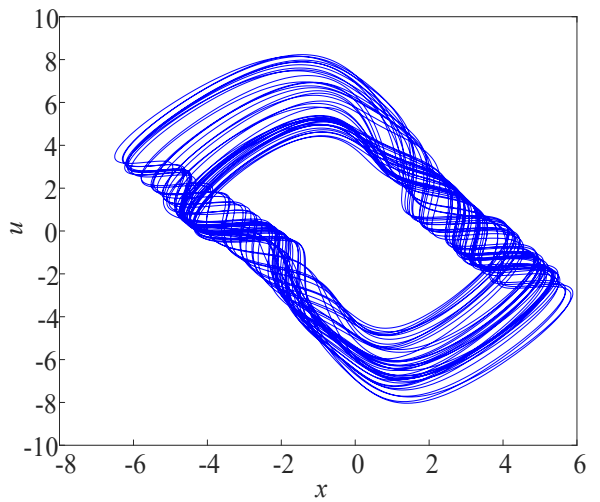

(a) $x-u$

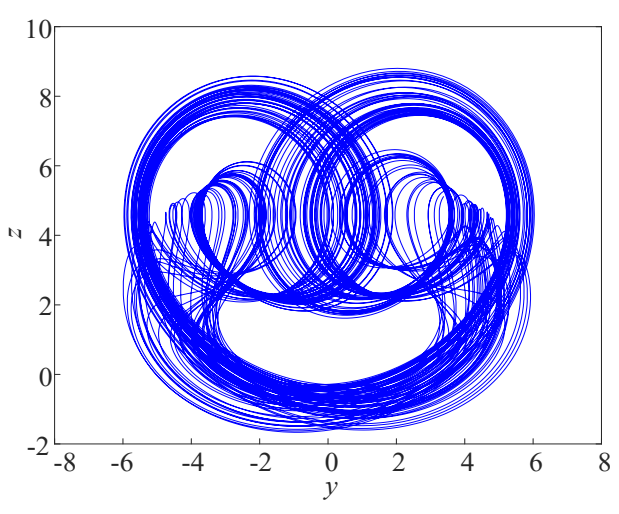

(b) $y-z$

Fig. 5 When $a=-10, b=-10, c=15$, chaotic attractor phase diagram

\subsection{Equilibrium point and stability}

According to the dynamical equations (6) of system, the divergence can be expressed as:

$$
\nabla V=\frac{\partial \dot{x}}{\partial x}+\frac{\partial \dot{y}}{\partial y}+\frac{\partial \dot{z}}{\partial z}+\frac{\partial \dot{u}}{\partial u}+\frac{\partial \dot{w}}{\partial w}=a-c-5-1,
$$

Let the system parameters $a=-10, b=-10, c=8$, the initial conditions are selected $(1,1,0,0$, 0 ), the divergence of the system is less than zero, so the system is dissipative, and the system might have chaotic attractors. Let the equations (6) of system be equal to zero, then the equilibrium point $\mathrm{E}(0,0,0,0,0)$ of the system can be obtained,according to the equilibrium point, the Jacobian matrix can be expressed as follows:

$$
J=\left[\begin{array}{ccccc}
a & a & 0 & 0 & 0 \\
-45 & 0 & 0 & 0 & 0 \\
0 & 0 & -c & 0 & 0 \\
0 & b & 0 & -5 & 0 \\
0 & 0 & 0 & 1 & 0
\end{array}\right],
$$

Its characteristic polynomial can be expressed as follows:

$$
P(\lambda)=\lambda^{5}+13 \lambda^{4}-460 \lambda^{3}+393.5593 \lambda^{2}+18000.0092 \lambda,
$$

According to the characteristic equation, the eigenvalues are $\lambda_{1}=0, \lambda_{2}=5, \lambda_{3}=-8, \lambda_{4}=-$ 
26.7945, and $\lambda_{5}=16.7945$. According to the Routh-Hurwitz criterion, the equilibrium point $\mathrm{E}$ $(0,0,0,0)$ is unstable.

\subsection{The impacts of parameters}

This system is a five-dimensional chaotic system. By combining the bifurcation diagram with Lyapunov exponent spectrum, the states of the system with different parameters can be obtained. In the following, the parameters a and $\mathrm{c}$ are put as variables, the initial conditions are selected $(1,1,0,0,0)$ the step size is $h=0.01$, the remaining parameters of the equation are fixed, and the different states of the system are observed by changing the parameters $a$ and $c$.

Take parameter $a \in[-10,1]$, let $b=-10, c=8$, Lyapunov exponent spectrum and bifurcation diagram can be expressed in Figure 6.It can be realized from Fig.6 that when $a=-8.25,-8.15$ and 0 , the maximum Lyapunov exponent L1 is equal to 0 , and the system behaves as a limit cycle state. At other times, the L1 is always greater than zreo,L2=0, and other Lyapunov exponents are less than 0 , so the system is a chaotic system.
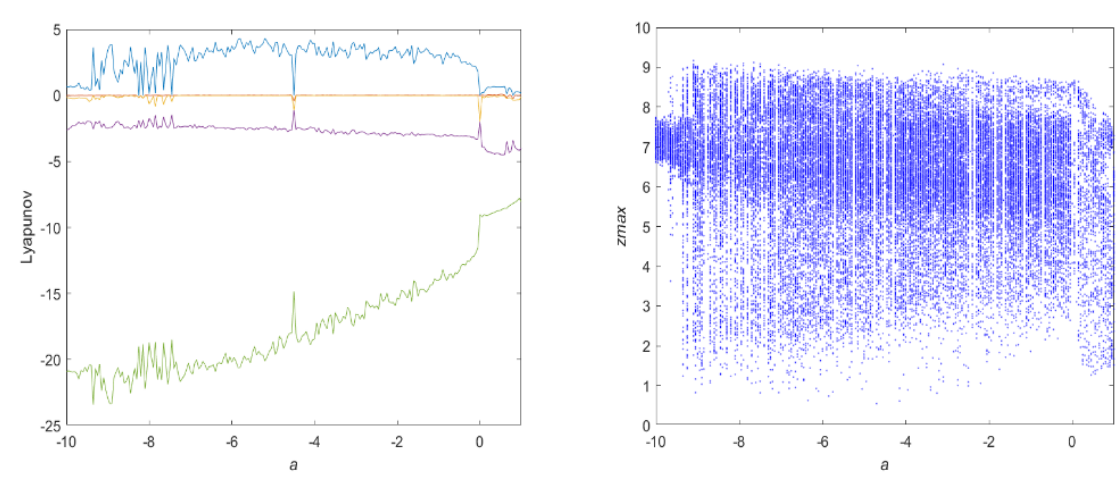

Fig. 6 Lyapunov exponential spectrum and bifurcation diagram of the system when $a$ changes

This result shows the phase diagram of $x-u$ when $c=8, b=-10$, fixed, $a=-8.25,-8.15,0$ and -8 , respectively. When $a=-8.25, a=-8.15$ and $a=0$, the phase diagram shows a limit cycle state. According to the Lyapunov exponent spectrum, there are four exponents less than 0 and one equal to 0 , and the system is in a periodic state; When $a=-8$, the phase diagram is chaotic. At this time, the other Lyapunov exponents of the system are less than $0, \mathrm{~L} 1>0$, and $\mathrm{L} 2=0$, which are in chaotic state. The analysis shows that this result is completely corresponding to the bifurcation diagram and Lyapunov exponent spectrum shown above. 


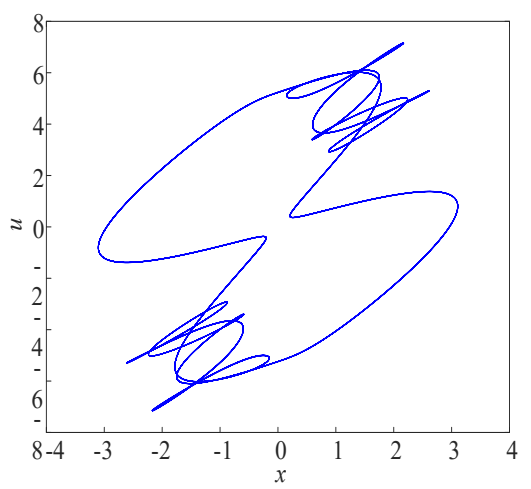

(a) $a=-8.25$

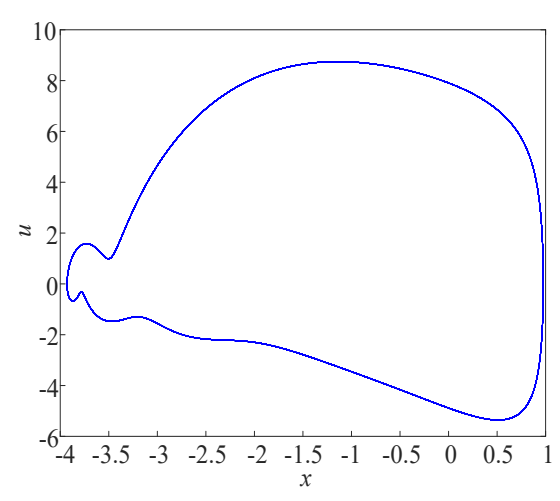

(c) $a=0$

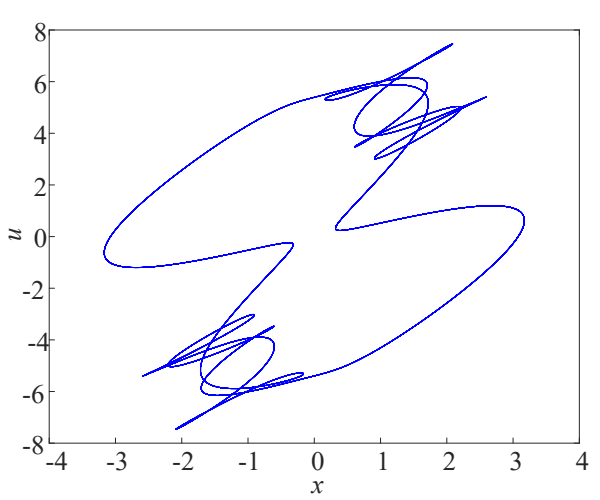

(b) $a=-8.15$

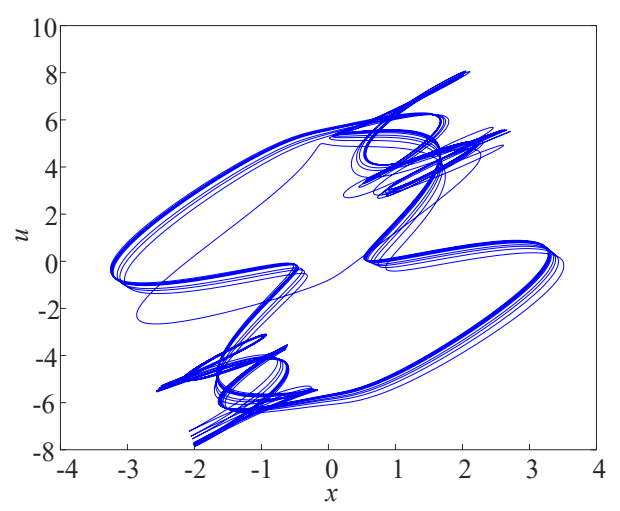

(d) $a=-8$

Fig. 7 The phase diagram of the system with different $a$

Take the $c \in[0,18], a=-10, b=-10$, and the Lyapunov exponent spectrum and bifurcation diagram under this condition are expressed in Figure 8. When $c \in[0,0.55]$, the $\mathrm{L} 1=0$, and the system behaves as a limit cycle. When $c=0.55$, chaos appears. When $c \in[0.55,8.85]$, the $\mathrm{L} 1>0$, and the other exponents is less than 0 , then the system shows chaotic state. Since $c \in[8.85$, 8.9], the maximum Lyapunov exponent $\mathrm{L} 1$ is equal to 0 , and the system behaves as a limit cycle. When $c \in[8.9,16.9]$, the $\mathrm{L} 1>0$, and the other exponents is less than 0 , and the system is in chaotic state. Since $c \in[16.9,18]$, the L1 is equal to zero and the other items are less than 0 , and the system behaves as a limit cycle state. 

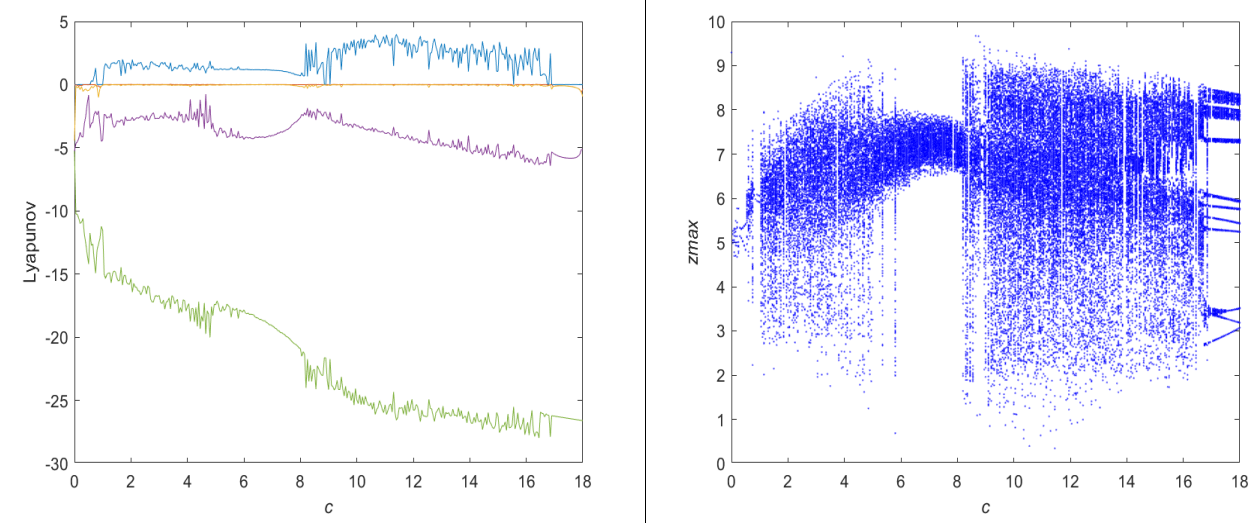

Fig. 8 Lyapunov exponential spectrum and bifurcation diagram of the system when $c$ changes

This result shows the phase diagram of $x-u$ when the parameters $a=-10, b=-10$, fixed and c are $0.45,5,8.85,15$ and 17.4 respectively. When $c=0.45, c=8.85$ and $c=17.4$, the phase diagram is a limit cycle. According to the Lyapunov exponent spectrum, there are four exponents of the system that are less than 0 , another is equal to 0 , and the system is in cycle state; When $c=5$ and $c=15$, the phase diagram is chaotic. At this time, three Lyapunov exponents of the system are less than 0 , one equal to zero and another greater than 0 , it means that it is chaotic system. The analysis shows that this result is completely corresponding to the bifurcation diagram and Lyapunov exponent spectrum shown above.

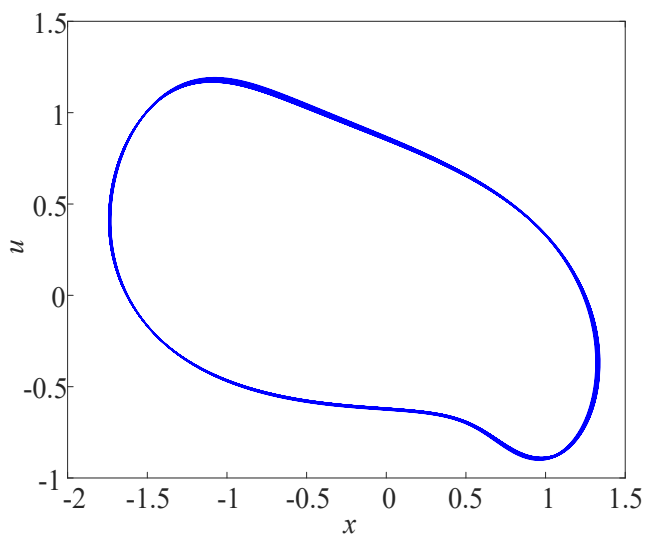

(a) $c=0.45$

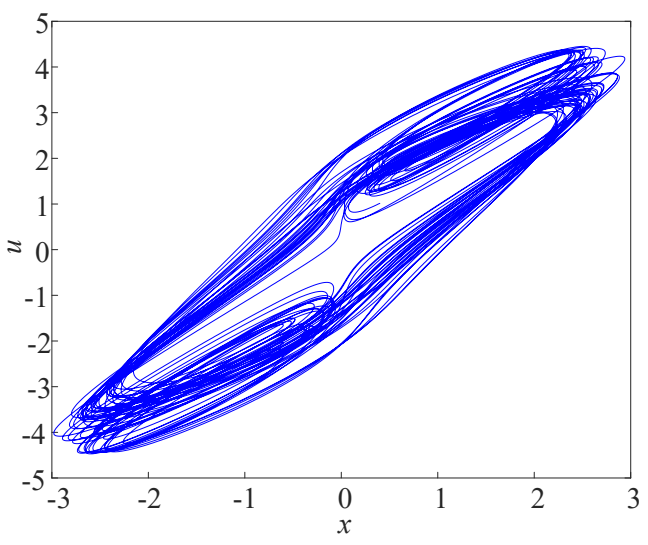

(b) $c=6$ 


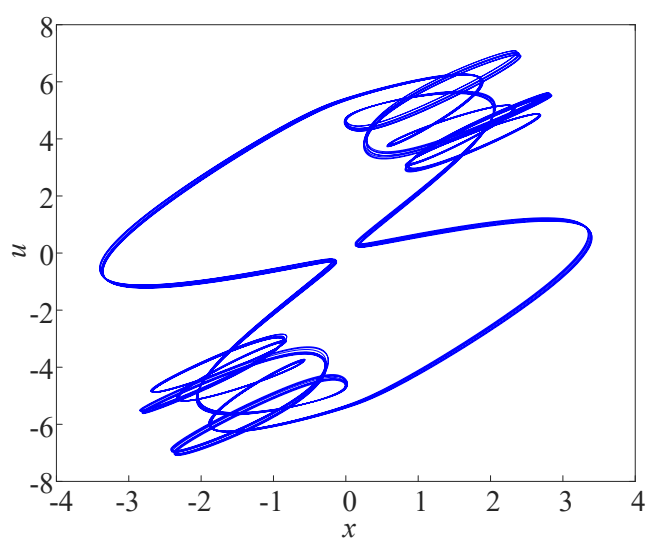

(b) $c=8.85$

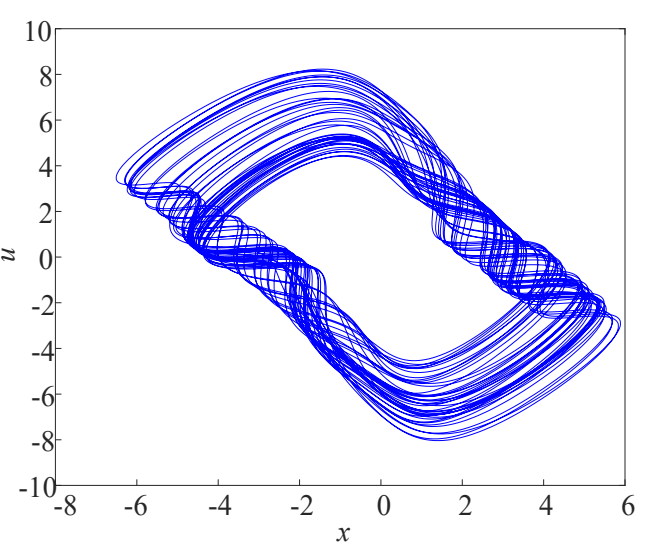

(d) $c=15$

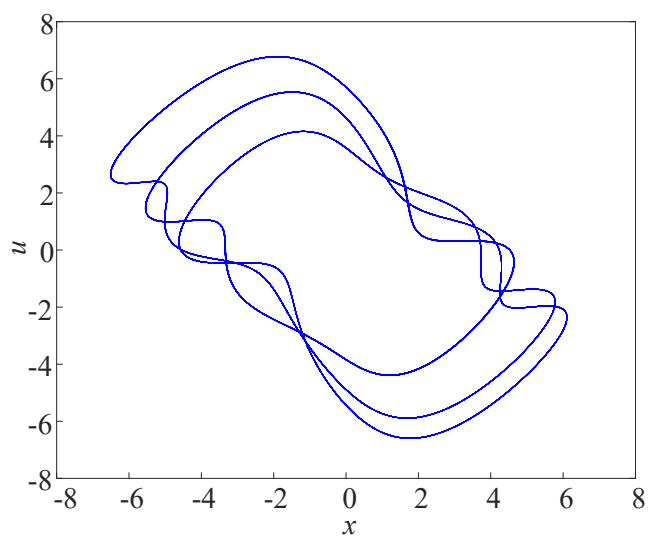

(e) $\quad c=17.4$

Fig.9 The phase diagram of the system with different $c$

\subsection{Complexity analysis}

The study of complexity involves various fields, and scholars in different fields have different understandings of complexity. So far, there is no unified concept of complexity. The complexity of chaotic system is to measure the degree of chaotic sequence approaching random sequence by using correlation algorithm. The greater the complexity, the closer the sequence is to the random sequence, the higher the security. In essence, the complexity of chaotic systems belongs to the study of chaotic dynamics.

Analysis of complexity includes behavioral complexityand structural complexity.Behavioral complexity refers to using some method to measure the probability of generating new patterns in a short time window from the chaotic sequence itself.The more likely a new pattern is to be generated, the more complex the sequence. At present, there are 
many algorithms to calculate the behavior complexity of chaotic pseudo-random sequences, and they are all based on Kolmogorov method and Shannon entropy. This kind of algorithm has fast calculation speed and accurate results. However, if the selected dimension is too high or the symbol space of pseudo-random sequences is too large, the calculation results will overflow or even get no results. The more balanced the energy spectrum distribution in the transform domain, the closer the original sequence is to the random signal, that is, the greater the complexity of the sequence. Combined with the concept of Shannon entropy, the corresponding spectral entropy can be calculated. The energy characteristics of this region are analyzed by using structural complexity, which is for the whole sequence rather than the local sequence. Therefore, compared with the behavior complexity algorithm, the results obtained are more global. In this paper, the complexity of the system are analyzed by using SE algorithm and $\mathrm{CO}$ algorithm.

SE algorithm mainly uses the Fourier transform, through the energy distribution in the Fourier transform domain, combined with Shannon entropy to obtain the spectral entropy value. The gist of $\mathrm{C} 0$ algorithm is to decompose the sequence into two parts, regular and irregular, and calculate the irregular part to get the result.

In this part, the complexity of the system is analyzed with parameter $c$ as a variable. When the parameters $c=8, b=-10$, fixed, and $a \in[-10,1]$, the simulation results are shown in Figure 10.

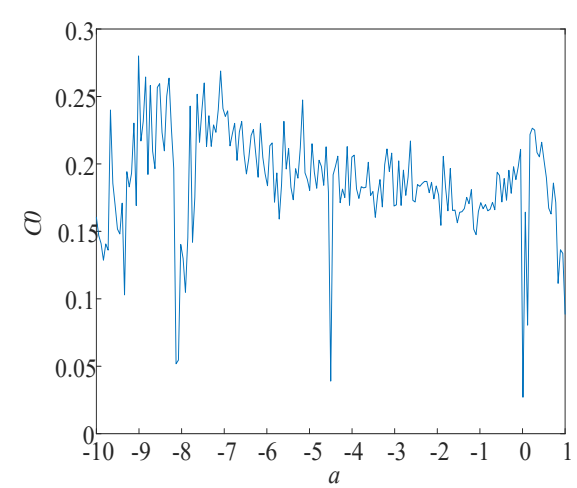

(a) C0 Complexity

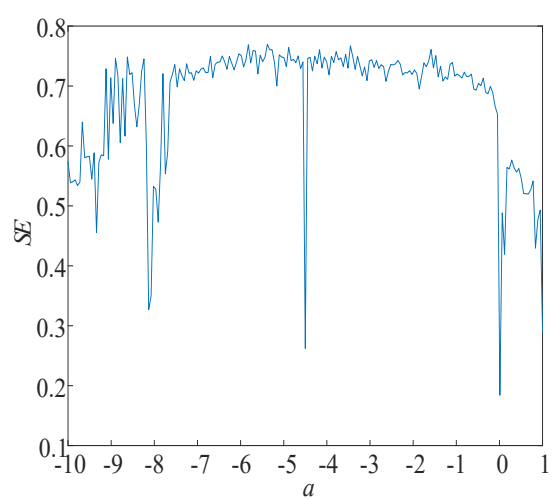

(b) SE Complexity

Fig. 10 Structural complexity curve with parameter $a$

By analyzing the complexity, it can be seen that SE algorithm and C0 algorithm have a high degree of synchronization. According to the image, when $a=-8.25,-8.15$ and 0 , the system shows a limit cycle state. On other scales, the system appears chaotic. By analyzing the $\mathrm{SE}$ complexity diagram and the $\mathrm{C} 0$ complexity diagram, it can be found that the complexity is 
at a low point when the system is in the periodic state, while the complexity increases significantly when the system enters the chaotic state. The results presented by the complexity are consistent with the Lyapunov exponential spectrum and bifurcation diagram as shown in Figure 8 .

\subsection{Coexistence of attractors}

Attractor coexistence is a peculiar phenomenon, which primarily emerges in some peculiar chaotic systems. It is very important in the study of nonlinear systems. Since the system parameters remain unchanged and the original values change, the orbits of the system tend to different states, such as point, quasi periodic, periodic or chaotic. In order to explore this special phenomenon, let $\mathrm{a}=-10, \mathrm{~b}=-10, \mathrm{c}=5$, with the change of initial value, we can observe the change of system state. The results of numerical simulation are shown in Figure 11. The blue part in the figure indicates that the initial value is $(1,2,3,4,10)$, and the red part indicates that the initial value is $(1,0,3,4,1)$.

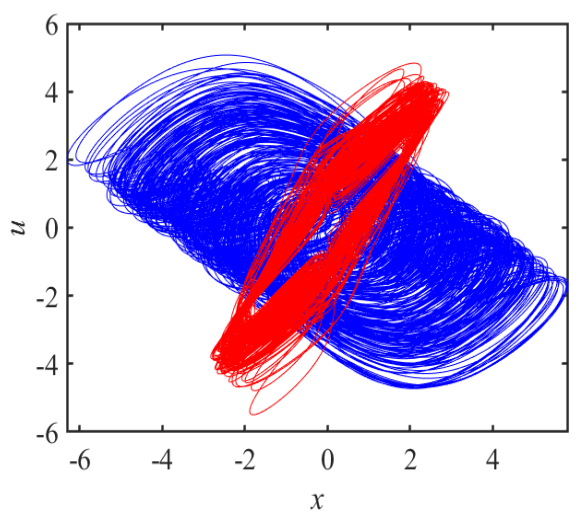

Figure 11. The diagram of attractor coexistence.

\section{Multisim simulation}

Because chaotic system is easily affected by external disturbance when it is implemented by analog circuit, it is difficult to control the relevant characteristic conditions accurately in the actual circuit. In this section, the system is simulated by using Multisim platform,and the realization of the system is proved.

The simulated electric circuit is illustrated in Figure 2, and the arguments of each component in the circuit are illustrated in Table 1 . The corresponding parameters are $a=-10$, $b=-10$, and $c=8$, and the image shown in Figure 12 is obtained. 


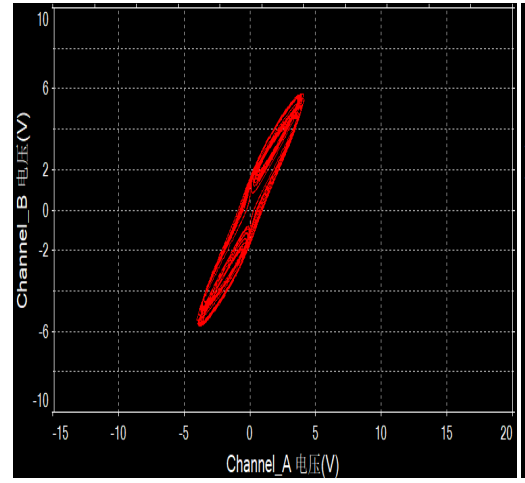

(a) $x-u$

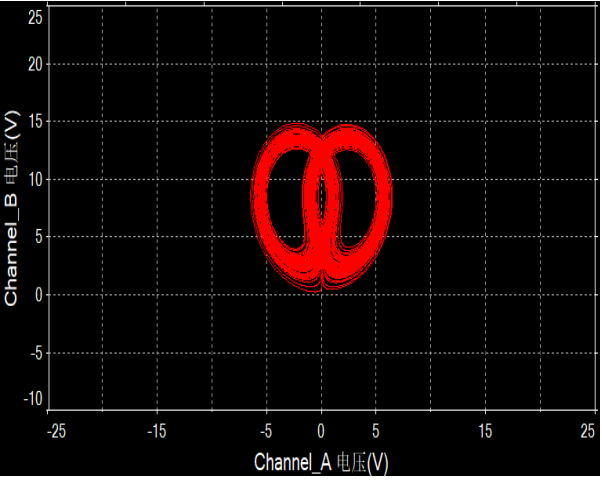

(b) $y-z$

Fig. 12. The phase diagram of Multisim simulation

\section{Conclusion}

In this article, we devise a newfangled Lorentz type chaotic circuit and introduce its dimensionless equation. Three different kinds of chaotic attractors are found in this chaotic system. By analyzing the Lyapunov exponential spectrum, bifurcation diagram and complexity of the system, it is found that the dynamic characteristics of the new system show a high degree of complexity and sensitivity with the change of system parameters. Through numerical simulation, we observe the special phenomenon of chaotic attractor coexistence. At the same time, some reasonable explanations are given for these phenomena. At last, the circuit simulation is obtained on Multisim platform to prove the consistency between the circuit simulation and the numerical simulation. Due to these rich dynamic behaviors, the novel Lorentz chaotic system has a good application prospect in information encryption and secure communication. Next, we are going to go further, and we will try to go further and explore the applications of new circuits.

\section{Acknowledgements}

This work is supported by Scientific Research Funding Project of the Educational Department of Liaoning Province, China (J2020051).

\section{References}

[1] Taher A A, Christos V, Gerodimos N A, et al. A Novel Chaotic System without Equilibrium: Dynamics, Synchronization, and Circuit Realization[J]. Complexity, 2017, 2017:1-11.

[2] Ma X , Mou J , Liu J , et al. A novel simple chaotic circuit based on memristor-memcapacitor[J]. 
Nonlinear Dynamics, 2020, 100(5).

[3]Chua, L.: Memristor-the missing circuit element. IEEE Trans. Circuit Theory 18(5), 507-519 (1971). https://doi. org/10.1109/TCT.1971.1083337

[4] Chua, L.O., Kang, S.M.: Memristive devices and systems. Proc. IEEE 64(2), 209-223 (1976)

[5] Ventra, M.D., Pershin, Y.V., Chua, L.O.: Circuit elements with memory: memristors, memcapacitors, and meminductors. Proc. IEEE 97(10), 1717-1724 (2009)

[6] Biolek, D., Biolkova, V.: Mutator for transforming memristor into memcapacitor. Electron. Lett. 46(21), 1428 (2010). https://doi.org/10.1049/el.2010.2309

[7] Volos, Christos, K, et al. Antimonotonicity, Crisis and Multiple Attractors in a Simple Memristive Circuit[J]. Journal of Circuits Systems \& Computers, 2018.

[8] Xia, Q., Robinett, W., Cumbie, M.W., Banerjee, N., Cardinali, T.J., Yang, J.J., Wu, W., Li, X., Tong, W.M., Strukov, D.B.: Memristor-CMOS hybrid integrated circuits for reconfigurable logic. Nano Lett. 9(10), 3640 (2009)

[9] Benkouider K , Bouden T , Halimi M . Dynamical Analysis, Synchronization and Circuit Implementation of a New Hyperchaotic System with Line Equilibrium[C]// 2019 6th International Conference on Control, Decision and Information Technologies (CoDIT). IEEE, 2019.

[10] Chua L O, Komuro M, Matsumoto T. The double scroll family[J]. 1986, 33(11):1072-1118.

[11] Lorenz, Edward N. Deterministic Nonperiodic Flow[J]. Journal of Atmospheric Sciences, 20(2):130-141.

[12] Pehlivan I , Uyaroglu Y . Simplified chaotic diffusionless Lorentz attractor and its application to secure communication systems[J]. Iet Communications, 2007, 1(5):1015-1022.

[13] Kurylev Y , Lassas M , Uhlmann G . Inverse problems for Lorentzian manifolds and non-linear hyperbolic equations[J]. Inventiones Mathematicae, 2018.

[14] Sundarapandian V , Karthikeyan R . Anti-Synchronization of Hyperchaotic Lorenz andHyperchaotic Chen Systems by Adaptive Control[J]. International Journal of Engineering Science \& Technology, 2012, 3(5):41-50.

[15] Hu K, Wang Y K, Dai H L, et al. Nonlinear and chaotic vibrations of cantilevered micropipes conveying fluid based on modified couple stress theory[J]. International Journal of Engineering Science, 2016, 105(Aug.):93-107.

[16] Tlidi M , Clerc M G . [Springer Proceedings in Physics] Nonlinear Dynamics: Materials, Theory and Experiments Volume 173 || Hyper-Chaotic and Chaotic Synchronisation of Two Interacting Dipoles[J]. 2016, 10.1007/978-3-319-24871-4(Chapter 20):261-272.

[17] Mahmoud E E, Alá A. Eshmawi. Secure communication and synchronizations in light of the stability theory of the hyperchaotic complex nonlinear systems[J]. Journal of Intelligent and Fuzzy Systems, 2019, 38(3):1-15. 
[18] Liu C S . A novel Lie-group theory and complexity of nonlinear dynamical systems[J]. Communications in Nonlinear Science \& Numerical Simulation, 2015, 20(1):39-58.

[19] He X , Li C , Huang J , et al. Generalized synchronization of arbitrary-dimensional chaotic systems[J]. Optik - International Journal for Light and Electron Optics, 2015, 126(4):454-459.

[20] Bao H , Hu A, Liu W . Bipolar Pulse-Induced Coexisting Firing Patterns in Two-Dimensional Hindmarsh-Rose Neuron Model[J]. International Journal of Bifurcation and Chaos, 2019, 29(01).

[21] Fang, Yuan, Guangyi, et al. Extreme multistability in a memristor-based multi-scroll hyper-chaotic system.[J]. Chaos, 2016.

[22] Chang H, Li Y, Chen G, et al. Extreme Multistability and Complex Dynamics of a Memristor-Based Chaotic System[J]. International Journal of Bifurcation and Chaos, 2020, 30(08):434-445.

[23] Bao B , Jiang T, Wang G, et al. Two-memristor-based Chua's hyperchaotic circuit with plane equilibrium and its extreme multistability[J]. Nonlinear Dynamics, 2017.

[24] Kengne J . Coexistence of Chaos with Hyperchaos, Period-3 Doubling Bifurcation, and Transient Chaos in the Hyperchaotic Oscillator with Gyrators[J]. International Journal of Bifurcation \& Chaos, 2015, 25(4):4285-762.

[25] Ling G , Ge M F , Hu F Y , et al. Coexistence of multi-attractor in small genetic regulatory networks[C]// 2020 39th Chinese Control Conference (CCC). 2020.

[26] Bao B , Xu L , Wu Z , et al. Coexistence of multiple bifurcation modes in memristive diode-bridge-based canonical Chua's circuit[J]. International Journal of Electronics, 2018:00207217.2018.1426122.

[27] Schaller G, Kiesslich G, Brandes T . Counting Statistics in Multi-stable Systems[J]. Physical review. B, Condensed matter, 2010, 81(20):2498-2502.

[28] Chen C , Chen J, Bao H, et al. Coexisting multi-stable patterns in memristor synapse-coupled Hopfield neural network with two neurons[J]. Nonlinear Dynamics, 2019, 95(10). 